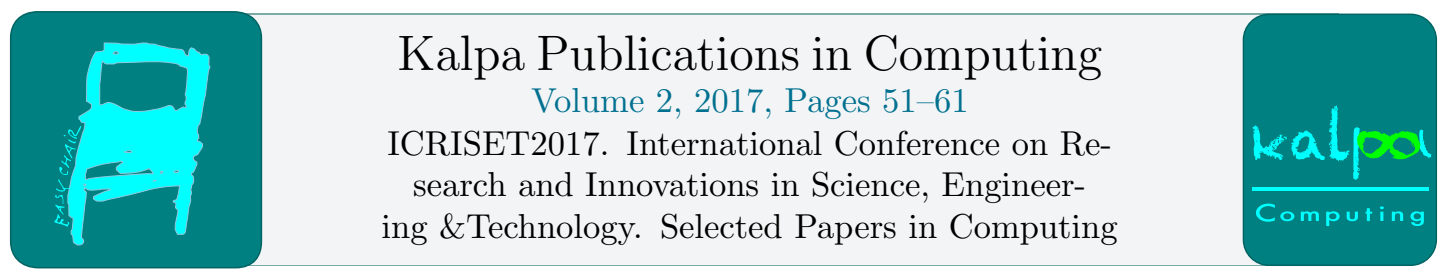

\title{
A Mathematical Model of Cocurrent Imbibition Phenomenon in Inclined Homogeneous Porous Medium
}

\author{
Mahendra A. Patel ${ }^{1}$ and N. B. Desai ${ }^{2}$ \\ 1 Government Engineering College, Gandhinagar-382028, Gujarat (India) \\ mahendraapatel@yahoo.co.in \\ 2 A. D. Patel Institute of Technology, New V. V. Nagar-388121, Gujarat (India) \\ drnbdesai@yahoo.co.in
}

\begin{abstract}
Spontaneous imbibition is the process in which the wetting phase is drawn into a porous medium by means of capillary force. Cocurrent and countercurrent spontaneous imbibitions are defined as wetting and non-wetting fluid flow in identical, and opposite directions respectively. The mathematical model is developed for cocurrent imbibition phenomenon in the inclined oil formatted homogeneous porous medium. An approximate analytical solution of the governing equation is derived by homotopy analysis method. The graphical and numerical solutions are discussed.
\end{abstract}

\section{Introduction}

One of the most important process in oil recovery is the spontaneous imbibition which is driven by capillary force. Such spontaneous imbibition may occur in the form of cocurrent imbibition or countercurrent imbibition. The direction of flow is the main difference between these two crucial mechanisms for imbibition. In cocurrent imbibition, the wetting and non-wetting phases flow in the same direction with the non-wetting phase being pushed out ahead of the wetting phase. In countercurrent imbibition, the wetting and non-wetting phases flow in the opposite directions. Imbibition in water-wet porous media is commonly considered as countercurrent imbibition $[3,5,7,13,20,24]$. When a porous medium is partially filled with wetting phase, oil recovery is dominated by cocurrent imbibition phenomenon, not countercurrent imbibition. In the oil recovery process, cocurrent imbibition is more efficient than countercurrent imbibition $[3,20,24]$.

Cocurrent imbibition phenomenon have been investigated by many authors with different viewpoints [3, 13, 20, 24]. Bourblaux and Kalaydjian [3] have discussed experimental study of cocurrent and countercurrent flows in natural porous media. Pooladi-Darvish and Firoozabadi [20] have studied the similarities and differences of cocurrent and countercurrent imbibition and pointed out the consequences for practical applications. Series solution is obtained for cocurrent imbibition during immiscible two-phase flow through porous media by Yadav and Mehta [24]. Exact integral solutions for the horizontal, unsteady flow of two viscous, incompressible fluids are derived by Mcwhorter and Sunada [13]. Homotopy perturbation method is used for solving 
the problem of cocurrent and countercurrent imbibition flow into vertical porous medium by Fazeli et al. [7].

During secondary oil recovery process, it is assumed that the water is injected into fractured oil formatted inclined homogeneous porous medium and cocurrent imbibition phenomenon occurs. It is also assumed that the macroscopic behavior of fingers is governed by statistical treatment. Thus only average cross sectional area occupied by fingers is taken into account, the size and shape of individual fingers are disregarded. The velocity of oil and the velocity of water are considered under gravitational effect and inclination effect. For the investigated flow system, the porosity and permeability of inclined homogeneous porous medium are assumed to be constants. The saturation of injected water $S_{w}(x, t)$ is then defined as the average cross-sectional area occupied by injected water at distance $x$ and time $t$.

In the current work, the mathematical model is developed for cocurrent imbibition phenomenon occurring during secondary oil recovery process. The mathematical formulation of cocurrent imbibition generates a one dimensional nonlinear partial differential equation. Homotopy analysis method is adopted to solve this equation with appropriate boundary conditions. The solution describes the saturation of injected water at distance $x$ and time $t$ for cocurrent imbibition phenomenon in inclined homogeneous porous medium.

\section{Mathematical Modelling}

\subsection{Fundamental equations}

During the injection process, two-phase immiscible and incompressible flow in porous medium is governed by the generalized Darcy's law for each phase as [2, 15, 21]:

$$
\begin{aligned}
V_{w} & =-\frac{k_{w}}{\delta_{w}} K\left(\frac{\partial P_{w}}{\partial x}+\rho_{w} g \sin \theta\right) \\
V_{o} & =-\frac{k_{o}}{\delta_{o}} K\left(\frac{\partial P_{o}}{\partial x}+\rho_{o} g \sin \theta\right)
\end{aligned}
$$

where $V_{w}$ and $V_{o}$ are the velocities of water and oil respectively, $k_{w}$ and $k_{o}$ are the relative permeabilities of water and oil respectively, $\delta_{w}$ and $\delta_{o}$ are the constant viscosities of water and oil respectively, $K$ is the permeability of the inclined homogeneous porous medium, $P_{w}$ and $P_{o}$ are the pressures of water and oil respectively, $\rho_{w}$ and $\rho_{o}$ are the constant densities of water and oil respectively, $g$ is the acceleration due to gravity, $\theta$ is the angle of inclination with porous matrix.

The law of conservation of mass for incompressible flow gives

$$
P \frac{\partial S_{w}}{\partial t}+\frac{\partial V_{w}}{\partial x}=0
$$

where $P$ is the porosity.

The sum of the velocities of injected water and native oil is the total velocity $V_{t}$ in cocurrent imbibition phenomenon [9]

$$
V_{w}+V_{o}=V_{t}
$$




\subsection{Standard relations}

The difference between pressures of oil and water is defined as the capillary pressure $\left(P_{c}\right)$. We assume that the capillary pressure is a function of phase saturation $[19,21]$ :

$$
P_{c}\left(S_{w}\right)=P_{o}-P_{w}
$$

Assume that the relationship between capillary pressure and phase saturation is of the form $[14]$

$$
P_{c}\left(S_{w}\right)=-\beta S_{w}
$$

where $\beta$ is a constant.

According to Scheidegger and Johnson [22], consider the analytical relationship between relative permeability and phase saturation as

$$
k_{w}=S_{w} \text { and } k_{o}=1-\alpha S_{w}
$$

where $\alpha$ ia a constant.

\subsection{Equation of motion for saturation}

Combining (1), (2) and (4), we get

$$
-\frac{k_{w}}{\delta_{w}} K\left(\frac{\partial P_{w}}{\partial x}+\rho_{w} g \sin \theta\right)-\frac{k_{o}}{\delta_{o}} K\left(\frac{\partial P_{o}}{\partial x}+\rho_{o} g \sin \theta\right)=V_{t}
$$

Using (5) in (8)

$$
\frac{k_{w}}{\delta_{w}} K\left(\frac{\partial P_{w}}{\partial x}+\rho_{w} g \sin \theta\right)+\frac{k_{o}}{\delta_{o}} K\left(\frac{\partial P_{c}}{\partial x}+\frac{\partial P_{w}}{\partial x}+\rho_{o} g \sin \theta\right)=-V_{t}
$$

Solving (9) for $\frac{\partial P_{w}}{\partial x}$

$$
\frac{\partial P_{w}}{\partial x}=-\left(K \frac{k_{w}}{\delta_{w}}+K \frac{k_{o}}{\delta_{o}}\right)^{-1}\left(K\left(\frac{k_{o}}{\delta_{o}} \rho_{o}+\frac{k_{w}}{\delta_{w}} \rho_{w}\right) g \sin \theta+K \frac{k_{o}}{\delta_{o}} \frac{\partial P_{c}}{\partial x}+V_{t}\right)
$$

Combining (1) and (10) results in

$$
V_{w}=-\frac{k_{w}}{\delta_{w}}\left(\frac{k_{w}}{\delta_{w}}+\frac{k_{o}}{\delta_{o}}\right)^{-1}\left(K \frac{k_{o}}{\delta_{o}}\left(\rho_{w}-\rho_{o}\right) g \sin \theta-K \frac{k_{o}}{\delta_{o}} \frac{\partial P_{c}}{\partial x}-V_{t}\right)
$$

The pressure of water can be expressed in the form

$$
P_{w}=\frac{P_{w}+P_{o}}{2}+\frac{P_{w}-P_{o}}{2}=\bar{P}-\frac{1}{2} P_{c}
$$

where $\bar{P}$ is the mean pressure which is constant, therefore (9) reduces to

$$
K\left(\frac{k_{w}}{\delta_{w}} \rho_{w}+\frac{k_{o}}{\delta_{o}} \rho_{o}\right) g \sin \theta+\frac{K}{2}\left(\frac{k_{o}}{\delta_{o}}-\frac{k_{w}}{\delta_{w}}\right) \frac{\partial P_{c}}{\partial x}=-V_{t}
$$


Therefore (11) implies

$$
V_{w}=\frac{K}{2} \frac{k_{w}}{\delta_{w}} \frac{\partial P_{c}}{\partial x}-K \frac{k_{w}}{\delta_{w}} \rho_{w} g \sin \theta
$$

Substituting (14) into (3), we get

$$
P \frac{\partial S_{w}}{\partial t}+\frac{\partial}{\partial x}\left[\frac{K}{2} \frac{k_{w}}{\delta_{w}} \frac{\partial P_{c}}{\partial x}-K \frac{k_{w}}{\delta_{w}} \rho_{w} g \sin \theta\right]=0
$$

Since $k_{w}=S_{w}$ and $P_{c}=-\beta S_{w}$, we have

$$
P \frac{\partial S_{w}}{\partial t}-\frac{K \beta}{2 \delta_{w}} \frac{\partial}{\partial x}\left[S_{w} \frac{\partial S_{w}}{\partial x}\right]-\frac{K \rho_{w} g \sin \theta}{\delta_{w}} \frac{\partial S_{w}}{\partial x}=0
$$

Using dimensionless variables

$$
X=\frac{x}{L}, \quad T=\frac{\beta K t}{2 \delta_{w} L^{2} P},
$$

(16) reduces to

$$
\frac{\partial S_{w}}{\partial T}=\frac{\partial}{\partial X}\left[S_{w} \frac{\partial S_{w}}{\partial X}\right]+A \frac{\partial S_{w}}{\partial X}
$$

where $A=\frac{2 L \rho_{w} g \sin \theta}{\beta}$ and $S_{w}(x, t)=S_{w}(X, T)$.

Eq. (17) is the nonlinear partial differential equation for the cocurrent imbibition phenomenon in the inclined homogeneous porous medium and $S_{w}(X, T)$ is the solution of this equation which represents the saturation of injected water at distance $X$ and time $T$.

We used following boundary conditions for solving (17):

$$
S_{w}(0, T)=0 \text { and } S_{w}(1, T)=\frac{2(1+T)}{5}
$$

\section{Solution by Homotopy Analysis Method}

The homotopy analysis method which was first proposed by Liao $[10,11]$ is an effective and powerful technique to obtain an approximate analytical solution of nonlinear differential equation. The homotopy analysis method has been widely applied to solve nonlinear partial differential equations $[4,6,8,12,16,17,18,23]$. Here we apply HAM to the nonlinear PDE (17). Consider the nonlinear partial differential equation

$$
\mathcal{N}[\phi(X, T ; q)]=0
$$

where $\mathcal{N}$ is a nonlinear operator.

We define a nonlinear operator

$$
\mathcal{N}[\phi(X, T ; q)]=\phi(X, T ; q) \frac{\partial^{2} \phi(X, T ; q)}{\partial X^{2}}+\left\{\frac{\partial \phi(X, T ; q)}{\partial X}\right\}^{2}+A \frac{\partial \phi(X, T ; q)}{\partial X}-\frac{\partial \phi(X, T ; q)}{\partial T}
$$


We choose the auxiliary linear operator

$$
\mathcal{L}[\phi(X, T ; q)]=\frac{\partial^{2} \phi(X, T ; q)}{\partial X^{2}} .
$$

According to the boundary conditions (18), we choose the initial approximation of $S_{w}(X, T)$ as

$$
S_{w_{0}}(X, T)=\frac{2\left(X^{2}+T X\right)}{5} .
$$

Let $q \in[0,1]$ denote the embedding parameter, $c_{0} \neq 0$ the convergence control parameter, $H(X, T) \neq 0$ an auxiliary function, $\mathcal{L}$ an auxiliary linear operator with the property $\mathcal{L}(f)=0$ when $f=0$. The so-called zeroth-order deformation equation is

$$
(1-q) \mathcal{L}\left[\phi(X, T ; q)-S_{w_{0}}(X, T)\right]=c_{0} q H(X, T) \mathcal{N}[\phi(X, T ; q)] .
$$

Thus when $q=0$ and $q=1$, we have

$$
\phi(X, T ; 0)=S_{w_{0}}(X, T) \text { and } \phi(X, T ; 1)=S_{w}(X, T) .
$$

Thus $\phi(X, T ; q)$ continuously deforms from the initial approximation $S_{w_{0}}(X, T)$ to the exact solution $S_{w}(X, T)$ of (17) as $q$ increases from 0 to 1 . Expanding $\phi(X, T ; q)$ in Maclaurin series with respect to $q$, we have the homotopy-Maclaurin series

$$
\phi(X, T ; q)=S_{w_{0}}(X, T)+\sum_{m=1}^{\infty} S_{w_{m}}(X, T) q^{m}
$$

where

$$
S_{w_{m}}(X, T)=\left.\frac{1}{m !} \frac{\partial^{m} \phi(X, T ; q)}{\partial q^{m}}\right|_{q=0}
$$

is the mth-order homotopy derivative of $\phi(X, T ; q)$. Assume that the auxiliary linear operator $\mathcal{L}$, the initial approximation $S_{w_{0}}(X, T)$, the convergence control parameter $c_{0}$ and the auxiliary function $H(X, T)$ are so properly chosen that the homotopy-Maclaurin series (25) converges at $q=1$, we have due to (24) the homotopy series solution

$$
S_{w}(X, T)=S_{w_{0}}(X, T)+\sum_{m=1}^{\infty} S_{w_{m}}(X, T) .
$$

Write $\overrightarrow{S_{w_{n}}}=\left\{S_{w_{0}}, S_{w_{1}}, \ldots, S_{w_{n}}\right\}$. Differentiating (23) $m$ times with respect to $q$, then setting $q=0$ and finally dividing them by $m$ !, we have the so-called high-order deformation equation

$$
\mathcal{L}\left[S_{w_{m}}(X, T)-\chi_{m} S_{w_{m-1}}(X, T)\right]=c_{0} H(X, T) \mathcal{R}_{m}\left(\overrightarrow{S_{w_{m-1}}}\right)
$$

subject to the boundary conditions

$$
S_{w_{m}}(0, T)=0 \text { and } S_{w_{m}}(1, T)=0, m \geq 1
$$

where

$$
\chi_{m}= \begin{cases}0 & \text { if } m \leq 1 \\ 1 & \text { if } m>1\end{cases}
$$


and

$$
\mathcal{R}_{m}\left(\overrightarrow{S_{w_{m-1}}}\right)=\sum_{i=0}^{m-1} S_{w_{i}} \frac{\partial^{2} S_{w_{m-1-i}}}{\partial X^{2}}+\sum_{i=0}^{m-1} \frac{\partial S_{w_{i}}}{\partial X} \frac{\partial S_{w_{m-1-i}}}{\partial X}+A \frac{\partial S_{w_{m-1}}}{\partial X}-\frac{\partial S_{w_{m-1}}}{\partial T}, m \geq 1 .
$$

For the sake of simplicity, assume $H(X, T)=1$. Then the solution of (28) reads

$$
S_{w_{m}}(X, T)=\chi_{m} S_{w_{m-1}}(X, T)+c_{0} \mathcal{L}^{-1}\left[\mathcal{R}_{m}\left(\overrightarrow{S_{w_{m-1}}}\right)\right]+C_{1} X+C_{2}
$$

where the coefficients $C_{1}$ and $C_{2}$ are determined by the boundary conditions (29). Hence

$$
\begin{aligned}
S_{w}(X, T) & =\frac{2 X^{2}}{5}+\frac{2 T X}{5}+c_{0}\left(-\frac{X}{75}-\frac{2 A X}{15}-\frac{4 T X}{25}-\frac{A T X}{5}-\frac{2 T^{2} X}{25}\right. \\
& \left.+\frac{A T X^{2}}{5}+\frac{2 T^{2} X^{2}}{25}-\frac{X^{3}}{15}+\frac{2 A X^{3}}{15}+\frac{4 T X^{3}}{25}+\frac{2 X^{4}}{25}\right)+c_{0}\left[-\frac{X}{75}\right. \\
& -\frac{2 A X}{15}-\frac{4 T X}{25}-\frac{A T X}{5}-\frac{2 T^{2} X}{25}+\frac{A T X^{2}}{5}+\frac{2 T^{2} X^{2}}{25}-\frac{X^{3}}{15}+\frac{2 A X^{3}}{15} \\
& +\frac{4 T X^{3}}{25}+\frac{2 X^{4}}{25}+c_{0}\left(-\frac{7 X}{375}-\frac{7 A X}{750}+\frac{A^{2} X}{30}-\frac{T X}{75}+\frac{A T X}{25}\right. \\
& +\frac{A^{2} T X}{30}+\frac{A T^{2} X}{75}-\frac{A X^{2}}{150}-\frac{A^{2} X^{2}}{15}-\frac{2 T X^{2}}{375}-\frac{2 A T X^{2}}{15}-\frac{A^{2} T X^{2}}{10} \\
& -\frac{8 T^{2} X^{2}}{125}-\frac{3 A T^{2} X^{2}}{25}-\frac{4 T^{3} X^{2}}{125}+\frac{8 X^{3}}{375}-\frac{A X^{3}}{50}-\frac{14 T X^{3}}{375}-\frac{2 A T X^{3}}{25} \\
& +\frac{A^{2} T X^{3}}{15}+\frac{8 A T^{2} X^{3}}{75}-\frac{4 T^{2} X^{3}}{125}+\frac{4 T^{3} X^{3}}{125}-\frac{A X^{4}}{30}+\frac{A^{2} X^{4}}{30}-\frac{T X^{4}}{25} \\
& \left.\left.+\frac{13 A T X^{4}}{75}+\frac{12 T^{2} X^{4}}{125}-\frac{13 X^{5}}{375}+\frac{26 A X^{5}}{375}+\frac{12 T X^{5}}{125}+\frac{4 X^{6}}{125}\right)\right]+\cdots
\end{aligned}
$$

is an approximate analytical expression of the solution of nonlinear partial differential equation (17) which represents the saturation of cocurrent imbibition phenomenon in inclined homogeneous porous medium.

\section{Results and Discussion}

The convergence of homotopy analysis solution is strongly dependent on convergence control parameter $c_{0}$. Many researchers have discussed the convergence of homotopy analysis solution using $c_{0}$-curve; for example, Darvishi and Khani [4] have obtained series solution of the foam drainage equation, Abbasbandy at al. [1] have discussed mathematical properties of $c_{0}$-curve in the frame work of the homotopy analysis method, Ghotbi at al. [8] have obtained the homotopy analysis solution of Richard's equation for unsaturated flow of transports in soils, Fariborzi and Naghshband [6] have discussed the convergence of homotopy analysis method to solve the Schrodinger equation with a power law nonlinearity, Patel and Desai [17] have obtained the solution of nonlinear partial differential equation of countercurrent imbibition phenomenon in inclined homogeneous porous medium.

The $c_{0}$-curve helps us to discover the valid region of $c_{0}$, which corresponds to the line segment almost parallel to the horizontal axis $[1,4,6,8,17,18]$. The BVPh 1.1, a Mathematica 
package [12] is used to plot the $c_{0}$-curves. To obtain the numerical and graphical representations of the solution, we assume that the value of constants are as $L=1, \rho_{w}=0.1, g=9.8, \beta=2$.

\subsection{Without inclination with porous matrix i.e. $\theta=0^{\circ}$.}

The homotopy analysis solution of cocurrent imbibition is derived for horizontal homogeneous porous medium and its convergence depends on $c_{0}$ which is chosen from $c_{0}$-curve. Fig. 1 shows the $c_{0}$-curve of $S_{w_{X X}}(0,0)$ for 30 th order approximation and $c_{0}=-0.1$ is chosen from this $c_{0}$-curve.

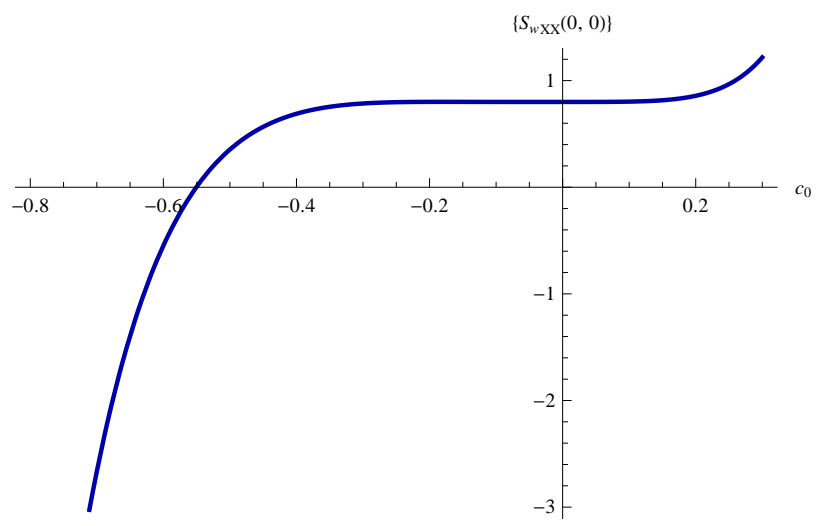

Fig. 1. The $c_{0}$-curve of $S_{w_{X X}}(0,0)$.

Table 1 indicates the numerical values of saturation of injected water for cocurrent imbibition phenomenon in horizontal homogeneous porous medium. The graph of saturation of injected water versus distance $X$ for fixed time $T=0.1,0.2, \ldots, 1$ is shown in Fig. 2.

Table 1: Numerical values of the saturation of injected water for $\theta=0^{\circ}$.

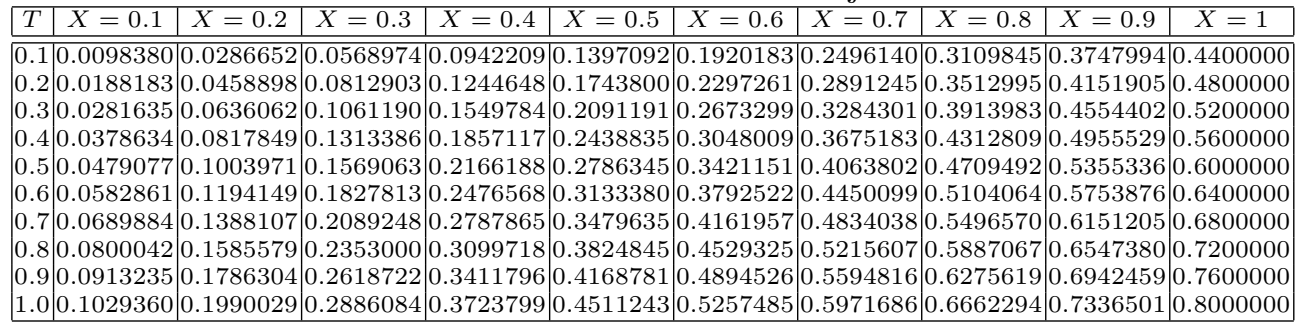




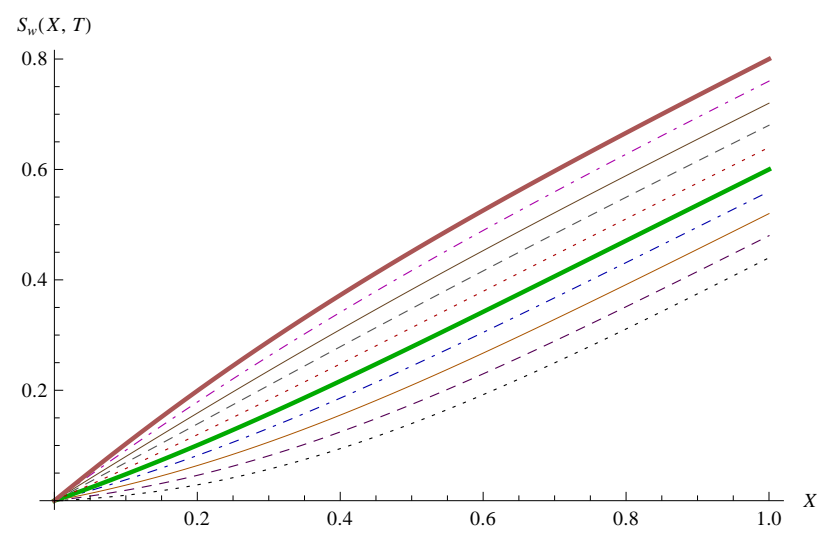

Fig. 2. Saturation of water $\mathrm{v} / \mathrm{s}$ distance $X$ for fixed time $T=0.1,0.2, \ldots, 1$.

\section{2 $\theta=5^{\circ}$ inclination with porous matrix.}

Fig. 3 shows the $c_{0}$-curve of $S_{w_{X X}}(0,0)$ for 30 th order approximation and the proper value of $c_{0}=-0.1$ chosen for convergent homotopy analysis solution of cocurrent imbibition in inclined $\left(\theta=5^{\circ}\right)$ homogeneous porous medium.

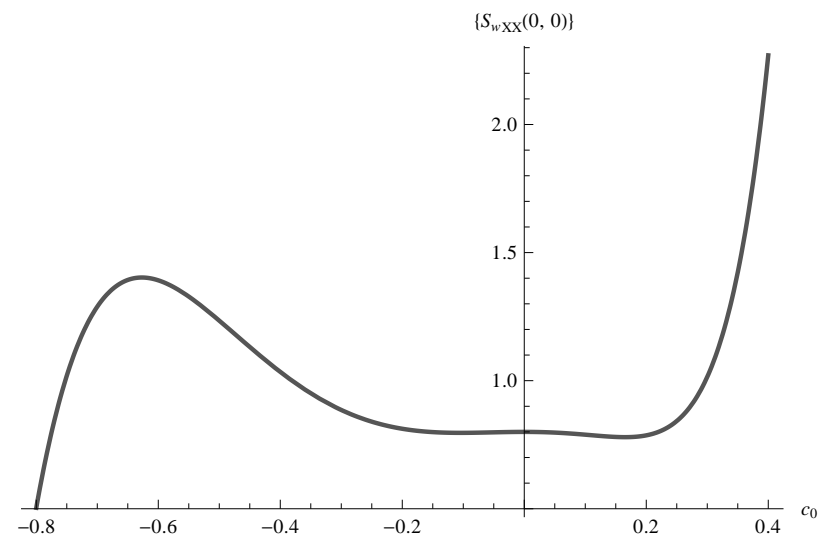

Fig. 3. The $c_{0}$-curve of $S_{w_{X X}}(0,0)$.

Table 2 indicates the numerical values of saturation of injected water for cocurrent imbibition phenomenon. The graph of saturation of injected water versus distance $X$ for fixed time $T=$ $0.1,0.2, \ldots, 1$ is given in Fig. 4 .

Table 2: Numerical values of the saturation of injected water for $\theta=5^{\circ}$.

\begin{tabular}{|l|l|l|l|l|l|l|l|l|l|l|}
\hline$T$ & $X=0.1$ & $X=0.2$ & $X=0.3$ & $X=0.4$ & $X=0.5$ & $X=0.6$ & $X=0.7$ & $X=0.8$ & $X=0.9$ & $X=1$ \\
\hline 0.1 & 0.0134242 & 0.0353242 & 0.0658915 & 0.1046419 & 0.1505559 & 0.2022832 & 0.2583616 & 0.3174084 & 0.3782527 & 0.4400000 \\
0.2 & 0.0229441 & 0.0533840 & 0.0912083 & 0.1357445 & 0.1859259 & 0.2404929 & 0.2981836 & 0.3578808 & 0.4186973 & 0.4800000 \\
0.3 & 0.0328201 & 0.0719041 & 0.1169061 & 0.1670467 & 0.2212909 & 0.2785331 & 0.3377509 & 0.3981061 & 0.4589871 & 0.5200000 \\
0.4 & 0.0430415 & 0.0908550 & 0.1429412 & 0.1985016 & 0.2566122 & 0.3163803 & 0.3770560 & 0.4380880 & 0.4991283 & 0.56000000 \\
0.5 & 0.0535977 & 0.1102079 & 0.1692718 & 0.2300657 & 0.2918555 & 0.3540153 & 0.4160945 & 0.4778315 & 0.5391275 & 0.60000000 \\
0.6 & 0.0644781 & 0.1299345 & 0.1958584 & 0.2616994 & 0.3269906 & 0.3914225 & 0.4548646 & 0.5173427 & 0.5789915 & 0.64000000 \\
0.7 & 0.0756722 & 0.1500076 & 0.2226633 & 0.2933660 & 0.3619913 & 0.4285900 & 0.4933666 & 0.5566289 & 0.6187269 & 0.68000000 \\
0.8 & 0.0871694 & 0.1704004 & 0.2496513 & 0.3250324 & 0.3968351 & 0.4655087 & 0.5316030 & 0.5956982 & 0.6583407 & 0.7200000 \\
0.9 & 0.0989593 & 0.1910873 & 0.2767889 & 0.3566684 & 0.4315026 & 0.5021724 & 0.5695778 & 0.6345590 & 0.6978398 & 0.7600000 \\
1.0 & 0.1110315 & 0.2120431 & 0.3040445 & 0.3882467 & 0.4659777 & 0.5385776 & 0.6072965 & 0.6732204 & 0.7372307 & 0.8000000 \\
\hline
\end{tabular}




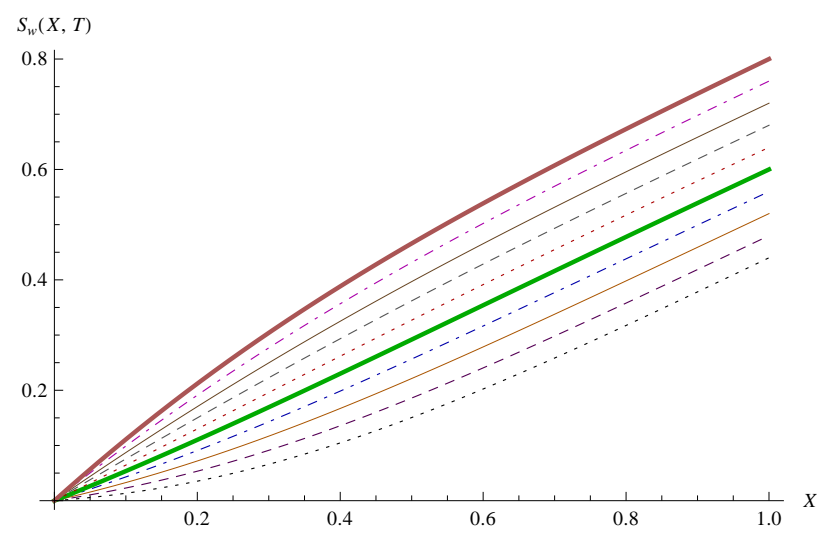

Fig. 4. Saturation of water $\mathrm{v} / \mathrm{s}$ distance $X$ for fixed time $T=0.1,0.2, \ldots, 1$.

\section{3 $\theta=10^{\circ}$ inclination with porous matrix.}

The homotopy analysis solution is obtained for cocurrent imbibition in inclined $\left(\theta=10^{\circ}\right)$ homogeneous porous medium. The $c_{0}$-curve of $S_{w_{X X}}(0,0)$ for 30 th order approximation is plotted; see Fig. 5. Here we choose proper value of $c_{0}=-0.1$.

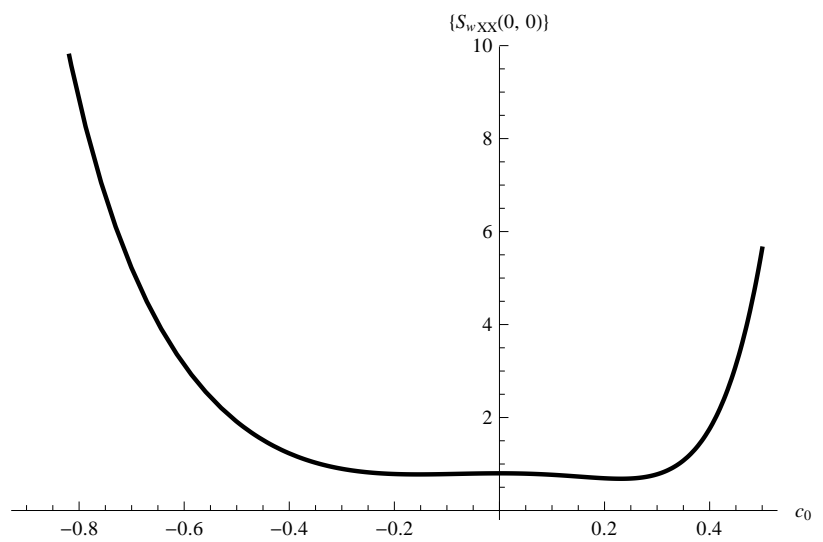

Fig. 5. The $c_{0}$-curve of $S_{w_{X X}}(0,0)$.

The numerical values of saturation of injected water for cocurrent imbibition phenomenon are obtained (see Table 3). Fig. 6 shows the graph of saturation of injected water versus distance $X$ for fixed time $T=0.1,0.2, \ldots, 1$.

Table 3: Numerical values of the saturation of injected water for $\theta=10^{\circ}$.

\begin{tabular}{|l|l|l|l|l|l|l|l|l|l|l|}
\hline$T$ & $X=0.1$ & $X=0.2$ & $X=0.3$ & $X=0.4$ & $X=0.5$ & $X=0.6$ & $X=0.7$ & $X=0.8$ & $X=0.9$ & $X=1$ \\
\hline 0.1 & 0.0171217 & 0.0421112 & 0.0749611 & 0.1150472 & 0.1612894 & 0.2123590 & 0.2668863 & 0.3236290 & 0.3815786 & 0.4400000 \\
0.2 & 0.0271826 & 0.0609980 & 0.1011812 & 0.1469785 & 0.1973249 & 0.2510391 & 0.3069950 & 0.3642431 & 0.4220696 & 0.4800000 \\
0.3 & 0.0375898 & 0.0803124 & 0.1277266 & 0.1790391 & 0.2332829 & 0.2894859 & 0.3468013 & 0.4045810 & 0.4623935 & 0.5200000 \\
0.4 & 0.0483324 & 0.1000247 & 0.1545544 & 0.2111850 & 0.2691290 & 0.3276812 & 0.3863026 & 0.4446497 & 0.5025581 & 0.5600000 \\
0.5 & 0.0593993 & 0.1201059 & 0.1816243 & 0.2433760 & 0.3048336 & 0.3656107 & 0.4254988 & 0.4844573 & 0.5425715 & 0.6000000 \\
0.6 & 0.0707798 & 0.1405281 & 0.2088981 & 0.2755751 & 0.3403706 & 0.4032635 & 0.4643923 & 0.5240130 & 0.5824417 & 0.6400000 \\
0.7 & 0.0824630 & 0.1612641 & 0.2363397 & 0.3077490 & 0.3757182 & 0.4406321 & 0.5029872 & 0.5633266 & 0.6221768 & 0.6800000 \\
0.8 & 0.0944381 & 0.1822875 & 0.2639152 & 0.3398674 & 0.4108576 & 0.4777116 & 0.5412894 & 0.6024085 & 0.6617847 & 0.7200000 \\
0.9 & 0.1066946 & 0.2035727 & 0.2915928 & 0.3719031 & 0.4457736 & 0.5144999 & 0.5793063 & 0.6412692 & 0.7012731 & 0.7600000 \\
1.0 & 0.1192218 & 0.2250951 & 0.3193426 & 0.4038318 & 0.4804535 & 0.5509968 & 0.6170462 & 0.6799197 & 0.7406496 & 0.8000000 \\
\hline
\end{tabular}




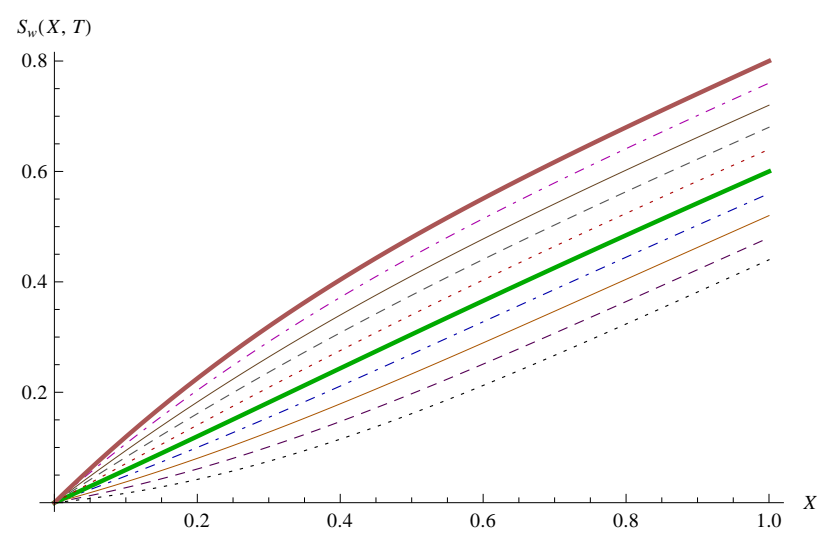

Fig. 6. Saturation of water $\mathrm{v} / \mathrm{s}$ distance $X$ for fixed time $T=0.1,0.2, \ldots, 1$.

\section{Conclusions}

We have discussed cocurrent imbibition phenomenon in inclined homogeneous porous medium and its mathematical model is derived. An approximate analytical solution is obtained for cocurrent imbibition phenomenon by homotopy analysis method. The solution satisfies both the boundary conditions. The numerical and graphical interpretations are given. The saturation of injected water increases when angle of inclination with porous matrix increases. We conclude that the saturation of injected water increases when the distance increases for given time $T$.

\section{Acknowledgments}

The authors are thankful to Government Engineering College, Sector-28, Gandhinagar for the financial support under TEQIP-II, encouragement and facilities.

\section{References}

[1] S. Abbasbandy, E. Shivanian, and K. Vajravelu. Mathematical properties of $\hbar$-curve in the frame work of the homotopy analysis method. Communications in Nonlinear Science and Numerical Simulation, 16, 4268-4275, 2011.

[2] J. Bear. Dynamics of fluids in porous media. American Elsevier Publishing Company, Inc., New York, 1972.

[3] B. J. Bourblaux and F. J. Kalaydjian. Experimental study of cocurrent and countercurrent flows in natural porous media. SPE Reservoir Engineering, Society of Petroleaum Engineers, 5(3), 361-368, 1990.

[4] M. T. Darvishi and F. Khani. A series solution of the foam drainage equation. Computers and Mathematics with Applications, 58, 360-368, 2009.

[5] N. B. Desai. The study of problems arises in single phase and multiphase flow through porous media. Ph.D. Thesis, South Gujarat University, Surat, India, 2002.

[6] M. A. Fariborzi Araghi and S. Naghshband. On convergence of homotopy analysis method to solve the schrodinger equation with a power law nonlinearity. International Journal of Industrial Mathematics, 5(4), 367-374, 2013. 
[7] H. Fazeli, R. Fathi, and A. Atashdehghan. Application of homotopy perturbation method to nonlinear equations describing cocurrent and countercurrent imbibition in fractured porous media. Journal of Chemical and Petroleum Engineering, University of Tehran, 46(1), 13-29, 2012.

[8] A. R. Ghotbi, M. Omidvar, and A. Barari. Infiltration in unsaturated soils - an analytical approach. Computers and Geotechnics, 38, 777-782, 2011.

[9] M. C. Leverett. Capillary behavior in porous solids. Transactions of the AIME, 142, 152-169, 1941.

[10] S. J. Liao. The proposed homotopy analysis technique for the solution of nonlinear problems. Ph.D. Thesis, Shanghai Jiao Tong University, Shanghai, China, 1992.

[11] S. J. Liao. Beyond perturbation: Introduction to the homotopy analysis method. Chapman and Hall/CRC Press, Boca Raton, 2003.

[12] S.J. Liao. Homotopy analysis method in nonlinear differential equations. Higher Education Press, Beijing and Springer-Verlag Berlin Heidelberg, 2012.

[13] D. B. Mcwhorter and D. K. Sunada. Exact integral solutions for two-phase flow. Water Resources Research, 26(3), 399-413, 1990.

[14] M. N. Mehta. Asymptotic expansions of fluid flow through porous media. Ph.D. Thesis, South Gujarat University, Surat, India, 1977.

[15] M. Muskat. The flow of homogeneous fluids through porous media. First edition, McGraw-Hill Book Company, Inc., New York and London, 1937.

[16] M. A. Patel and N. B. Desai. An approximate analytical solution of nonlinear differential equation arising in fluid flow through homogeneous porous media. International Journal of Innovative Research in Science, Engineering and Technology, 4(8), 7655-7662, 2015.

[17] M. A. Patel and N. B. Desai. Homotopy analysis solution of countercurrent imbibition phenomenon in inclined homogeneous porous medium. Global Journal of Pure and Applied Mathematics, 12(1), 1035-1052, 2016.

[18] M. A. Patel and N. B. Desai. Homotopy analysis method for fingero-imbibition phenomenon in heterogeneous porous medium. Nonlinear Science Letters A: Mathematics, Physics and Mechanics, 8(1), 90-100, 2017.

[19] D. W. Peaceman. Fundamentals of numerical reservoir simulation. Elsevier Scientific Publishing Company, New York, 1977.

[20] M. Pooladi-Darvish and A. Firoozabadi. Cocurrent and counterurrent imbibition in a water-wet matrix block. Society of Petroleaum Engineers Journal, 5(1), 3-11, 2000.

[21] A. E. Scheidegger. The physics of flow through porous media. revised edition, University of Toronto Press, Toronto, 1960.

[22] A. E. Scheidegger and E. F. Johnson. The statistically behaviour of instabilities in displacement process in porous media. Canadian Journal of Physics, 39(2), 326-334, 1961.

[23] K. Vajravelu and R. A. Van Gorder. Nonlinear flow phenomena and homotopy analysis: Fluid flow and heat transfer. Higher Education Press, Beijing and Springer-Verlag Berlin Heidelberg, 2012.

[24] S. R. Yadav and M. N. Mehta. Analytical approximate expression for cocurrent imbibition during immiscible two-phase flow through porous media. Mathematical Problems in Engineering, Hindawi Publishing Corporation, Article ID 638409, 1-6, 2014. 